\title{
Review Article \\ Holding the Inflammatory System in Check: TLRs and Their Targeted Therapy in Asthma
}

\author{
Zhiyong Dong, ${ }^{1}$ Lingxin Xiong, ${ }^{1}$ Weijie Zhang, ${ }^{2}$ Peter G. Gibson, ${ }^{3}$ Ting Wang, \\ Yanjiao Lu, ${ }^{1}$ Guoqiang Wang, ${ }^{1}$ Hui Li, ${ }^{2}$ and Fang Wang ${ }^{1}$ \\ ${ }^{1}$ Department of Pathogen Biology, Basic Medical College, Jilin University, Changchun 130021, China \\ ${ }^{2}$ Department of Respiratory Disease, Jilin Provincial People's Hospital, Changchun 130021, China \\ ${ }^{3}$ Department of Respiratory and Sleep Medicine, John Hunter Hospital, Newcastle, NSW 2305, Australia \\ Correspondence should be addressed to Fang Wang; wf@jlu.edu.cn
}

Received 24 February 2016; Accepted 13 April 2016

Academic Editor: Eda Holl

Copyright (C) 2016 Zhiyong Dong et al. This is an open access article distributed under the Creative Commons Attribution License, which permits unrestricted use, distribution, and reproduction in any medium, provided the original work is properly cited.

\begin{abstract}
Inflammation is a complex biological response to detrimental stimuli and can be a double-edged sword. Inflammation plays a protective role in removing pathogenic factors, but dysregulated inflammation is associated with several major fatal diseases such as asthma, cancer, and cardiovascular diseases. Asthma is a complex heterogenous disease caused by genetic and environmental factors. TLRs are the primary proteins associated with the innate and adaptive immune responses to these fatal factors and play an important role in recognizing pathogen-associated molecular patterns (PAMPs) and damage-associated molecular patterns (DAMPs), which initiates the downstream immune response. Due to the complex TLRs cascade and nowadays unsuccessful control in asthma, new studies are focused on TLRs and other potential targets in TLR cascade to minimize airway inflammation.
\end{abstract}

\section{Introduction}

Inflammation is a complex host response to detrimental stimuli including tissue injury, microbial infection, and irritant exposure. It is classically characterized by redness, swelling, heat, pain, and tissue dysfunction [1]. When inflammation involves mucosal surfaces, there are accompanying mucus hypersecretions and epithelial shedding. Inflammation plays a protective role in the body in negating pathogenic factors such as microbial infections and oxidative stress and is a healing process enabling repair of damaged tissue [2]. On the contrary, persistence of inflammation with overproduction of cytokines by immune cells including macrophages, neutrophils, eosinophils, dendritic cells, mast cells, natural killer cells, and structural cells such as endothelial cells, mucosal epithelial cells, and fibroblasts can be harmful. Dysregulated inflammation is associated with several diseases including asthma, cancer, cardiovascular disease, autoimmune diseases, and metabolic disease.

Asthma is a complex heterogeneous disease associated with local tissue chronic inflammation of the airway and is characterized by variable and recurring symptoms (including wheezing, coughing, chest tightness, and shortness of breath), reversible airflow obstruction, airway remodeling, and airway hyperresponsiveness. According to Chung [3], asthma is ranked as the 14th most important chronic disease worldwide regarding the prevalence, extent, and duration of disability and affects 334 million individuals of all ages, resulting in 90 and 170 deaths per million in female and male individuals, respectively. In addition, asthma causes a heavy economic burden for the government and individuals. For example, in Europe, total cost per patient ranges from $£ 509$ for controlled asthma to $£ 2281$ for uncontrolled asthma [4]. Asthma is caused by a complex and incompletely understood combination of genetic (polymorphisms of multiple genes) and environmental (such as respiratory infections and particulates PM2.5) factors, which induce an immune response via the infiltration of inflammatory cells into the airway and consequent cytokine release. Emerging evidence shows that Toll-like receptors (TLRs) are associated with the inflammatory response and chronic airway inflammation in asthma [5]. TLRs are a subgroup of pattern recognition 
receptors (PRRs) that are expressed by cells of the innate immune system and that sense two classes of molecules such as pathogen-associated molecular patterns (PAMPs) and damage-associated molecular patterns (DAMPs), which then initiates the downstream immune cascades.

Many previous studies have focused on the discovery, structure, and roles of TLR family members and related signaling pathways in airway diseases, but few studies emphasize TLR expression in asthma, especially in the different phenotypes. This review will highlight the roles of TLR members in airway inflammation and their association with the pathogenesis of distinct asthma phenotypes and in addition will discuss the potential for TLR-targeted therapies in the treatment of asthma.

\section{TLRs Family and Related Signal Pathways}

Toll-like receptors (TLRs) are a class of single, transmembrane, and noncatalytic proteins named PRR and are expressed on specific immune cells (i.e., macrophages and dendritic cells) as well as nonimmune cells (e.g., epithelial, fibroblast, and endothelial cells) [6]. TLRs bind to and recognize endogenous molecules named DAMPs (e.g., structurally conserved components of microbes) and exogenous molecules that are named PAMPs (e.g., viral and bacterial products). Additionally, after the recognition by TLRs, downstream cascades are initiated. TLRs are involved in the initiation of innate immune responses and play a protective role against microbial infections. Once microbes invade physical barriers such as the skin or intestinal tract mucosa, TLRs on the cellular surface respond to microbial membrane materials (e.g., lipids and lipoproteins) and intracellular TLRs recognize microbial nucleic acids to initiate a host response [7].

So far, a total of 10 TLR genes in humans (TLR1-TLR10) and 12 (TLR1-TLR9 and TLR11-TLR13) in mice have been discovered. The 10 TLRs family members in humans are categorized into two subgroups. The first subgroup that recognizes the components of microbial membranes includes TLR1, TLR2, TLR4, TLR5, TLR6, and TLR10 of humans and TLR11 and TLR12 of mice and is primarily expressed on the cell surface [8]. The second subgroup that responds to microbial nucleic acids includes TLR3, TLR7, TLR8, and TLR9 and is expressed intracellularly in vesicles (e.g., lysosomes, endosomes, and the endoplasmic reticulum). TLR signaling is divided into two distinct signaling pathways, that is, the myeloid differentiation factor 88- (MyD88-) dependent and Toll/IL-1 receptor-domain containing adapter-inducing interferon- $\beta$ - (TRIF-) dependent pathway. Both pathways are involved in innate immunity. MyD88 and TRIF bind independently to TLRs, resulting in the production of cytokines such as TNF- $\alpha$, IL- $1 \beta$, IL-6, and type I IFNs [9].

2.1. MyD88 Pathway. MyD88 possesses an amino- (N-) terminal death domain (DD), a shorter linker sequence, and a carboxy- (C-) terminal Toll/interleukin-1 receptor (TIR) domain. MyD88 also has an intermediate domain (ID) that interacts with IL-1R-associated kinases 4 (IRAK4) in TLR signaling $[10,11]$. MyD88-dependent signaling is used by all TLRs except TLR3. The knockout of MyD88 in mice showed no responses to the ligands of TLR family members including TLR2, TLR4, TLR5, TLR7, and TLR9, indicative of the key role of MyD88 in TLRs-mediated inflammatory responses [12-16].

2.2. TRIF Pathway. TRIF is a large protein containing 712 amino acids in humans and directly binds to TLR3 and indirectly binds to TLR4 via connection with another adaptor protein, TRIF-related adaptor molecule (TRAM) [10]. The knockout of TRIF in mice triggers defective expression of IFN- $\beta$ production and IFN-related genes that are mediated by TLR3 and TLR4, although early-phase activation of NF$\kappa \mathrm{B}$ and TLR4-mediated activation of MyD88 pathway were observed [17]. Similarly, TRIF was confirmed to have a key role in the induction of inflammatory mediators contributing to antiviral innate immune responses via MyD88independent signaling that is mediated by both TLR3 and TLR4 [18].

\section{What Is Asthma?}

Asthma is a common heterogeneous disease characterized by chronic airway inflammation and is defined by recurring respiratory symptoms (such as wheezing, cough, shortness of breath, and chest tightness) that vary over time and in intensity, as well as by airflow obstruction according to GINA report [19]. Asthma causes a serious global health threat to patients of all age groups and is increasing in many countries in its prevalence, especially among children. Some countries have experienced a significant decline in hospitalizations and mortality from asthma; however, asthma still imposes a heavy burden on public health systems and on society through productivity decreases.

Due to both exposures (such as allergen and microbial infection) and treatment, there is heterogeneity in the inflammatory response in asthmatic airway. Wang et al. [20] previously categorized asthma into four phenotypes such as neutrophilic, eosinophilic, mixed granulocytic, and paucigranulocytic asthma according to inflammatory cell counts in induced sputum. Individualized precise diagnosis and treatment based on inflammatory phenotypes are now advocated because of limitations on the premise of current management of asthma. Individualized therapy is the customization of health care tailored to the individual and uses previously infeasible technologies based primarily on recent cluster analyses, molecular phenotyping, biomarkers, and differential responses to therapies, distinguishing a given patient from other patients with similar clinical presentations $[21,22]$. Nowadays, the mainstay of asthma treatment is daily long acting $\beta_{2}$ agonists and inhaled corticosteroids (LABA/ICS) [3]. Maintenance treatment with LABA/ICS relieves asthma symptoms and reduces the frequency of exacerbations; however, there are limits in treatment options for people who do not gain control on combination LABA/ICS [23]. Targeted therapies at IgE, interleukin-4 (IL-4), IL-4 receptor, IL-5, IL-13, tumor necrosis factor- $\alpha$, and CRTh2 are new treatment paradigms for asthma [24]. Emerging studies demonstrate that TLRs-targeted therapies potentially play a 


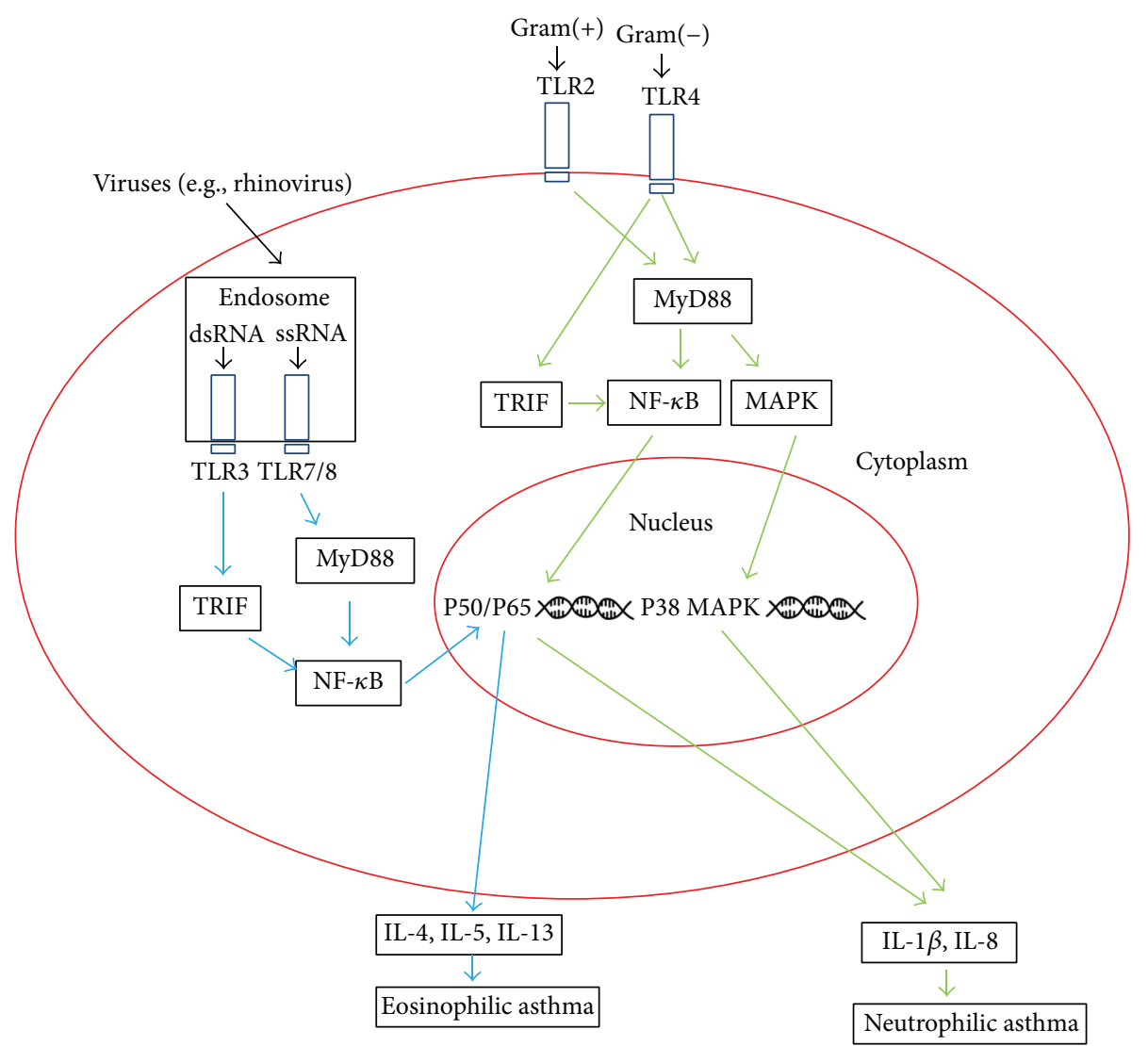

FIGURE 1: Schematic overview of TLR signaling pathway in neutrophilic and eosinophilic asthma. Gram-negative and Gram-positive bacteria as well as respiratory viruses (e.g., rhinovirus) are detected by TLRs. Subsequently, TLR3 and TLR7/8 trigger TRIF and MyD88 pathways, respectively, followed by the transcription of NF- $\kappa$ B in nucleus and the production of IL-4, IL-5, and IL-13, inducing eosinophilic asthma. TLR2 and TLR4 induce MyD88 and MyD88 as well as TRIF cascades, respectively, followed by the transcriptions of MAPK and NF- $\kappa$ B into nucleus, triggering the release of IL-1 $\beta$ and IL- 8 and the onset of neutrophilic asthma. MAPK: mitogen-activated protein kinase; MyD88: myeloid differentiation primary-response gene 88 ; NF- $\kappa$ B: nuclear factor- $\kappa \mathrm{B}$; TLR: Toll-like receptor; TRIF: Toll/IL-1R (TIR) domain containing adaptor protein inducing IFN- $\beta$.

key role in effectively controlling airway inflammation in asthma.

\section{TLRs in Asthma}

4.1. TLR2 and TLR4 in Neutrophilic Asthma. The role of adaptive immune responses in asthma is well studied and involves $\mathrm{T}$ helper type 2 lymphocyte activation by allergen, accompanied by eosinophilic airway inflammation. The innate immune system is also associated with the pathogenesis of asthma and the onset of inflammation in the airway. Simpson first discovered that an upregulation of the innate immune receptors TLR2 and TLR4 as well as proinflammatory cytokines IL- 8 and IL- $1 \beta$ was involved in neutrophilic asthma [25]. TLR2 plays an important role in recognizing Gram-positive bacteria and TLR4 is responsible for the detection of Gram-negative bacteria through their microbial components such as lipopolysaccharides (LPS) (Figure 1) [8].

4.2. TLR7 and Eosinophilic Asthma. TLR7 is intracellularly expressed on the surface of airway epithelia and airway smooth muscle as well as innate immune cells (such as macrophages, natural killer cells, and dendritic cells) [26, 27] and plays a significant role in the pathogenesis of autoimmune disorders such as Systemic Lupus Erythematosus (SLE) and in the regulation of antiviral immune responses [28]. TLR7 recognizes single-stranded RNA, a common molecular component to respiratory viruses, resulting in regulating downstream interferon production and the activation of Th1 antiviral responses [28]. TLR7 exhibits its antiviral activity in combination with TLR8, the homologue of TLR7 that also recognizes single-stranded viral RNA (Figure 1).

TLR7 plays an important role in reduction of airway inflammation, promoting Thl responses in immune cells, reversing airway hyperresponsiveness, and preventing airway remodeling. Airway inflammation is essential to the pathogenesis of asthma and is triggered by respiratory viral infections and inhaled allergen, leading to the activation of $\mathrm{T}$ helper 2 (Th2) cell differentiation and the secretion of Th2 cytokines such as IL-4, IL-5, and IL-13 [29]. IL5 matures eosinophils in the bone marrow and, together with chemokines such as eotaxins, promotes recruitment of eosinophils into the airways, resulting in local eosinophilic 
inflammation [30]. TLR7 stimulation suppresses eosinophilic airway inflammation in a variety of animal models of asthma through reducing Th2 cytokines such as IL- 4 and IL-5 as well as eotaxin in the lung [31] and IgE [32]. On the other hand, IL-5 induced airway eosinophilia can act as negative regulator of TLR7 expression and antiviral responses [30]. The role of TLR7 is not limited to Th 2 responses; besides, it is involved in Th1 responses in immune cells. TLR7 activation promotes the reduction of Th2 cells and the enhancement of Th1 cells, which results in increases in Thl-cytokine release and decrease in IgE production [33-35], exhibiting the immunomodulatory activity of TLR7 in maintaining Th2/Th1 balance. IL-13 is responsible for inducing airway hyperreactivity (AHR) and mucus production in eosinophilic asthma [36]. TLR7 stimulation ameliorates ovalbumin-induced AHR when animals are treated with TLR7 agonists. A number of emerging studies suggest that the suppression of AHR involves NF- $\kappa \mathrm{B}$ and p38 MAP intracellular signaling and is dependent on iNKT cells and IFN- $\gamma$ production $[26,37]$; however, in vivo investigation on the mechanism of AHR amelioration remains incomplete. Additionally, TLR7 ligand prevents chronic irreversible asthmatic airway remodeling including smooth muscle proliferation and goblet cell hyperplasia $[38,39]$.

\subsection{TLR Genetic Polymorphisms and Asthma. Genetic poly-} morphisms in TLRs may be responsible for individual susceptibility and severity of asthma. Genetic diversity in specific alleles determines the differences in susceptibility to a specific disease to some extent [40]. Polymorphisms in the TLR4 gene affect sensitivity to allergens [41, 42]. Zhang et al. [42] discovered a harmful effect of the TT homozygote allele in the TLR4 gene rs1927914 on the forced expiratory volume in 1s $\left(\mathrm{FEV}_{1}\right)$, implicating impaired lung function. Additionally, the AA homozygote genotype and A allele in Asp299 Gly of the TLR4 gene may correlate with a reduced asthma risk, as indicated by the association between TLR4 polymorphisms and the development of asthma in the study by Tizaoui et al. [43]. In addition to TLR4, variants of the TLR2 gene were reported to have some association with childhood asthma in Caucasians [44], and TLR2 polymorphism affects the asthma risk and lung function [45]. It has been shown that variants in the TLR7/8 genes as well as the TLR10 gene showed no significant association in some alleles despite the relevance between other polymorphisms in the TLR10 gene and asthma [42, 45-48]. In terms of TLR1 and TLR5, studies on the association between genetic polymorphisms and the development of asthma have not been reported. Future investigations should emphasize TLR genetic variants such as haplotype analysis and gene-environmental interaction [43].

4.4. TLR and Viral Infection. Viral infection is a common acute trigger of asthma and exacerbation of asthma. Approximately $80 \%$ of asthma exacerbations are caused by respiratory viral infection $[49,50]$. The PRRs in the detection of viral infection include TLR7 and TLR8 which detect single-stranded RNA and TLR3, retinoic acid-inducible gene I (RIG I), and melanoma differentiation associated gene 5 (MDA5) that are activated by double-stranded RNA. TLR7 expression is associated with the severity of the disease [51]. Airway cells from asthmatic patients are vulnerable to viral infection due to impaired innate antiviral responses compared to healthy subjects. This vulnerability is triggered by aberrant production of type I IFN, an antiviral cytokine [51]. TLR7 deficiency was discovered in alveolar macrophages from severe asthmatic and affected the interferon responses to rhinovirus infection. In the same study, the abnormal expression of the three microRNAs such as miR-150, miR-152, and miR-375 was the trigger of TLR7 deficiency. When these miRs were blocked, this resulted in restored TLR7 expression and augmented interferon responses to rhinovirus infection, indicating that TLR7 is associated with the vulnerability of asthmatic subjects [51]. In addition to this finding, in vivo research shows that a lack of TLR7 signaling in a rhinovirusinduced asthma exacerbation leads to reduced IFN production and exaggerated Th2-driven inflammation, suggesting the role of TLR7 signaling in rhinovirus-induced asthma exacerbation [30]. Other investigations support this finding. Bronchoalveolar lavage (BAL) cells from nonsevere asthma possess a deficient IFN response to rhinovirus infection [52, 53]; additionally, TLR7 dysfunction was shown in asthmatic peripheral blood mononuclear cells [54]. TLR3 also detects double-stranded RNA genome of respiratory virus which represents the replication of RNA viruses and protects the host by the induction of inflammatory responses including type I IFN production and activation of NK cells and cytotoxic T lymphocytes [55]. In an investigation by Parsons et al. [56], although no difference in the expression of TLR3 was observed, primary bronchial epithelial cells (pBECs) from asthmatics demonstrated an ineffective innate immune response following RV infection, with impaired type I and type III interferon responses to the infection. In addition, RV infection of healthy pBECs triggered a robust upregulation of TLR3, while inhibition of TLR3 signaling leads to a marked inhibition of both type I and type III interferon responses.

4.5. TLR9 and Asthma. TLR9 is intracellularly expressed in the immune cells such as B lymphocytes, monocytes, and plasmacytoid dendritic cells and detects unmethylated CpG motifs in microbial DNA molecules [57]. In allergic asthma subjects, TLR9 expression on plasmacytoid dendritic cells and TLR9-induced responses are upregulated by IL25 that originates from airway epithelial cells [58]. In an in vivo investigation in severe asthma, Duechs et al. [59] discovered that TLR9 activation significantly reduced some features of the asthmatic phenotype such as a reduction in eosinophil influx and IgE levels in serum. The same study also observed a decreased release of cytokines such as IL4 , IL-5, IFN- $\gamma$, IL-1 $\beta$, and IL-12, indicative of enhanced Th1 response, suggesting that TLR9 activation may suppress the Th2 response via promoting a Th1 response. Similarly, a Th1 response induced by the exposure to CpG DNA opposes a Th2 response in a murine model of asthma [60]. TLR9 is also involved in the inhibition of airway remodeling [61-64], suggesting a potential protective role of TLR9 in asthma. This was evaluated in in vivo models where TLR9 activation was found to be associated with a reduction in antigen-induced respiratory allergic responses $[65,66]$, suggesting that TLR9 
ligands could be used as prophylactic and therapeutic agents in asthma [67]. However, TLR9 targeted treatment was not found to be efficacious in preexisting severe allergic inflammation in the airway, in either animal experiments or clinical trials $[68,69]$. The role of TLR9 agonists in asthma requires further evaluation.

\section{TLRs Targeted Therapeutics}

5.1. Effect of TLR Agonists in Asthma. The typical treatment for asthma and asthma exacerbations includes inhaled corticosteroids for their ability of enhancing $\beta$-adrenergic responses and repressing inflammation in airways [70]. Nevertheless, in the treatment of severe asthma, corticosteroids are ineffective in alleviating symptoms, probably because oxidative stress as well as subsequent DNA damage leads to decreased activity of transcriptional corepressors such as histone deacetylase-2 (HDAC-2) [8]. Recently, TLRs agonists have been considered as agents in controlling asthma. TLRs agonists can be categorized into cellular surface TLRs agonists and intracellular TLRs agonists based on the distribution of TLRs. Cell surface TLRs sense structural components of microbia ranging from Gram-positive bacteria to Gramnegative bacteria and some respiratory viruses in the onset and development of asthma and asthma exacerbation [67]. Targeting TLR4 to treat asthma is based on the activation of TLR4 as an adjuvant in allergy vaccines to induce tolerance and inhibition of TLR4 expression. TLR4 agonists such as $\mathrm{MPL}^{\circledR}$ (monophosphoryl lipid A) seem to work effectively as allergy vaccines due to overexpression of TLR4 in asthmatic patients [8]. Another cell surface TLRs agonist is Pam3CSK 4 that acts as a synthetic TLR2 agonist and exhibits antiasthmatic effects by reducing Th2 cytokine release, AHR, IgE levels, airway inflammation, and asthmatic symptoms in animal models of asthma [67]. Intracellular TLRs agonists such as TLR7/TLR8 agonists have also been evaluated in asthma. Resiquimod is a typical TLR7/TLR8 agonist and in vivo suppresses AHR as well as airway remodeling in asthma [31, 39, 71-73]. In addition, this drug was also found to suppress both Th1 and Th2 cytokine production in the lungs of experimental animals and decrease lung eosinophilia, goblet cell hyperplasia, and IgE levels [39, 67, 71]. Many other agents that target TLRs have been found to control airway inflammation, eosinophilia, and AHR in distinct animal models of allergic inflammatory diseases [67]. It is obvious that in the future a wide variety of TLR agonists are likely to be evaluated as effective asthma controllers. On the contrary, future emphasis should be on the side effects of TLR agonists, especially on asthmatic children due to a lack of investigation on allergic children. Nowadays clinical trials are mainly conducted in adults, and besides uncertain targeting of the immature immunity in children as well as timing, dosage, and patient selection regarding the formulation to best employ TLRs agonists still needs further studies, which may hinder wider application of TLRs agonists.

5.2. Effect of Corticosteroid on TLR Expression. Corticosteroids are the most effective agents in inflammation management in asthma, and classical corticosteroids such as budesonide are recommended by guidelines for asthma treatment [74]. When inhaled corticosteroids (ICS) were introduced into asthma management, symptom control of asthma and lung function were improved, and exacerbations and asthma-related mortality decreased [19]. Corticosteroids influence TLRs and can upregulate TLR4 expression in vivo in peripheral blood mononuclear cells from asthmatic patients [75]. In addition to this finding, after in vitro stimulation with LPS, the production of both TNF- $\alpha$ and IFN- $\gamma$ in PBMC supernatant was significantly increased by oral corticosteroids [75]. Similarly, Pace et al. [76] reported that TLR4 and TLR2 expression were increased in Treg lymphocytes from allergic asthmatic subjects after budesonide treatment compared to healthy controls, providing further understanding of the action mechanism of budesonide on the control of inflammation in asthma. Furthermore, an increased level of IL-10 and decreased level of IL- 6 and TNF- $\alpha$ were observed after budesonide administration, confirming the modulatory potential of budesonide in immune responses to allergic subjects.

\section{Conclusions}

The invasion of antigens into airways causes the activation of PRRs such as TLRs in response to PAMPs. TLRs play an important role in the detection of invading pathogens by the innate immune system, and a total of 10 TLRs family members have been discovered in humans (TLR1TLR10). TLRs induce the activation of MyD88 and TRIF signaling pathways, resulting in the production of inflammatory mediators via the NF- $\kappa \mathrm{B}$ pathway. Different pathogens trigger distinct immune activation of TLRs. TLR2 plays an important role in recognizing Gram-positive bacteria and TLR4 is responsible for the detection of Gram-negative bacteria, leading to the production of cytokines such as IL$1 \beta$ and IL- 8 and to the infiltration of neutrophils in asthmatic airways. In addition, TLR7 senses single-stranded viral RNA which inhibits Th2 immune responses and eosinophilic asthma, and TLR9 detects unmethylated CpG motifs in microbial DNA molecules in the development of asthma and asthma exacerbation. Furthermore, genetic polymorphisms affect the susceptibility and severity of asthma, making effective control of airway inflammation in asthma more complex. Nowadays, corticosteroid therapy is commonly used for asthma treatment, and some findings confirmed the modulatory role of corticosteroid in the mediation of TLR expression in asthmatic subjects. Combination therapy of corticosteroid and TLRs agonists may be potentially more effective in controlling inflammation in asthmatics compared to the traditional treatment by corticosteroid. However, the timing, dosage, patient selection, and many other questions regarding the formulation to best employ TLRs agonists remain unclear, and future work needs to address these difficulties in order to hold airway inflammation in check in asthma.

\section{Competing Interests}

The authors declare that they have no competing interests. 


\section{Acknowledgments}

This work was supported by Training Program of Scientific and Technical Innovative Talent (Grant no. 201205014), Science and Technology Development Plan of Jilin Province of China (Grant no. 20150519015JH), and Bethune B Plan of Jilin University (Grant no. 2012218).

\section{References}

[1] O. Takeuchi and S. Akira, "Pattern recognition receptors and inflammation,” Cell, vol. 140, no. 6, pp. 805-820, 2010.

[2] R. Medzhitov, "Origin and physiological roles of inflammation," Nature, vol. 454, no. 7203, pp. 428-435, 2008.

[3] K. F. Chung, "Targeting the interleukin pathway in the treatment of asthma," The Lancet, vol. 386, no. 9998, pp. 1086-1096, 2015.

[4] S. Accordini, A. G. Corsico, M. Braggion et al., "The cost of persistent asthma in Europe: an international population-based study in adults," International Archives of Allergy and Applied Immunology, vol. 160, no. 1, pp. 93-101, 2013.

[5] S. Phipps, C. E. Lam, P. S. Foster, and K. I. Matthaei, “The contribution of toll-like receptors to the pathogenesis of asthma," Immunology and Cell Biology, vol. 85, no. 6, pp. 463-470, 2007.

[6] S. Goulopoulou, C. G. McCarthy, and R. C. Webb, “Toll-like receptors in the vascular system: sensing the dangers within," Pharmacological Reviews, vol. 68, no. 1, pp. 142-167, 2016.

[7] J. Jang, H. W. Shin, J. M. Lee, H. Lee, E. Kim, and S. H. Park, "An overview of pathogen recognition receptors for innate immunity in dental pulp," Mediators of Inflammation, vol. 2015, Article ID 794143, 12 pages, 2015.

[8] L. Zuo, K. Lucas, C. A. Fortuna, C. Chuang, and T. M. Best, "Molecular regulation of toll-like receptors in asthma and COPD," Frontiers in Physiology, vol. 6, article 312, 2015.

[9] V. Piras and K. Selvarajoo, "Beyond MyD88 and TRIF pathways in Toll-like receptor signaling," Frontiers in Immunology, vol. 5, article 70, 2014.

[10] K. B. Narayanan and H. H. Park, "Toll/interleukin-1 receptor (TIR) domain-mediated cellular signaling pathways," Apoptosis, vol. 20, no. 2, pp. 196-209, 2015.

[11] J. H. Lee, L. C. Wang, H. H. Yu, Y. T. Lin, Y. H. Yang, and B. L. Chiang, "Type I IL-1 receptor (IL-1RI) as potential new therapeutic target for bronchial asthma," Mediators of Inflammation, vol. 2010, Article ID 567351, 7 pages, 2010.

[12] T. Kawai, O. Adachi, T. Ogawa, K. Takeda, and S. Akira, "Unresponsiveness of MyD88-deficient mice to endotoxin," Immunity, vol. 11, no. 1, pp. 115-122, 1999.

[13] O. Takeuchi, K. Takeda, K. Hoshino, O. Adachi, T. Ogawa, and S. Akira, "Cellular responses to bacterial cell wall components are mediated through MyD88-dependent signaling cascades," International Immunology, vol. 12, no. 1, pp. 113-117, 2000.

[14] H. Häcker, R. M. Vabulas, O. Takeuchi, K. Hoshino, S. Akira, and $\mathrm{H}$. Wagner, "Immune cell activation by bacterial CpG-DNA through myeloid differentiation marker 88 and tumor necrosis factor receptor-associated factor (TRAF)6," The Journal of Experimental Medicine, vol. 192, no. 4, pp. 595-600, 2000.

[15] F. Hayashi, K. D. Smith, A. Ozinsky et al., "The innate immune response to bacterial flagellin is mediated by Toll-like receptor5," Nature, vol. 410, no. 6832, pp. 1099-1103, 2001.

[16] H. Hemmi, T. Kaisho, O. Takeuchi et al., "Small-antiviral compounds activate immune cells via the TLR7 MyD88-dependent signaling pathway," Nature Immunology, vol. 3, no. 2, pp. 196200, 2002.

[17] M. Yamamoto, S. Sato, H. Hemmi et al., "Role of adaptor TRIF in the MyD88-independent toll-like receptor signaling pathway," Science, vol. 301, no. 5633, pp. 640-643, 2003.

[18] K. Hoebe, X. Du, P. Georgel et al., "Identification of Lps2 as a key transducer of MyD88-independent TIR signalling," Nature, vol. 424, no. 6950, pp. 743-748, 2003.

[19] Global Initiative for Asthma, "GINA report, Global Stragety for Asthma Management and Prevention," Documents/Resources, GINA, April 2015, http://ginasthma.org/wp-content/uploads/ 2016/01/GINA_Report_2015_Aug11-1.pdf.

[20] F. Wang, X. Y. He, K. J. Baines et al., "Different inflammatory phenotypes in adults and children with acute asthma," European Respiratory Journal, vol. 38, no. 3, pp. 567-574, 2011.

[21] A. Agusti, E. Bel, M. Thomas et al., "Treatable traits: toward precision medicine of chronic airway diseases," European Respiratory Journal, vol. 47, no. 2, pp. 410-419, 2016.

[22] M. W. Pijnenburg and S. Szefler, "Personalized medicine in children with asthma," Paediatric Respiratory Reviews, vol. 16, no. 2, pp. 101-107, 2015.

[23] K. M. Kew and K. Dahri, "Long-acting muscarinic antagonists (LAMA) added to combination long-acting beta -agonists $_{2}$ and inhaled corticosteroids (LABA/ICS) versus LABA/ICS for adults with asthma," Cochrane Database of Systematic Reviews, no. 1, Article ID CD011721, 2016.

[24] J. B. Bice, E. Leechawengwongs, and A. Montanaro, "Biologic targeted therapy in allergic asthma," Annals of Allergy, Asthma and Immunology, vol. 112, no. 2, pp. 108-115, 2014.

[25] J. L. Simpson, T. V. Grissell, J. Douwes, R. J. Scott, M. J. Boyle, and P. G. Gibson, "Innate immune activation in neutrophilic asthma and bronchiectasis," Thorax, vol. 62, no. 3, pp. 211-218, 2007.

[26] A.-K. Ekman, M. Adner, and L.-O. Cardell, "Toll-like receptor 7 activation reduces the contractile response of airway smooth muscle," European Journal of Pharmacology, vol. 652, no. 1-3, pp. 145-151, 2011.

[27] I. Ioannidis, F. Ye, B. McNally, M. Willette, and E. Flaño, "Toll-like receptor expression and induction of type I and type III interferons in primary airway epithelial cells," Journal of Virology, vol. 87, no. 6, pp. 3261-3270, 2013.

[28] M. G. Drake, E. H. Kaufman, A. D. Fryer, and D. B. Jacoby, "The therapeutic potential of Toll-like receptor 7 stimulation in asthma," Inflammation \& Allergy-Drug Targets, vol. 11, no. 6, pp. 484-491, 2012.

[29] S. T. Holgate, G. Roberts, H. S. Arshad, P. H. Howarth, and D. E. Davies, "The role of the airway epithelium and its interaction with environmental factors in asthma pathogenesis," Proceedings of the American Thoracic Society, vol. 6, no. 8, pp. 655-659, 2009.

[30] L. Hatchwell, A. Collison, J. Girkin et al., “Toll-like receptor 7 governs interferon and inflammatory responses to rhinovirus and is suppressed by IL-5-induced lung eosinophilia," Thorax, vol. 70, no. 9, pp. 854-861, 2015.

[31] M. J. Duechs, C. Hahn, E. Benediktus et al., “TLR agonist mediated suppression of allergic responses is associated with increased innate inflammation in the airways," Pulmonary Pharmacology and Therapeutics, vol. 24, no. 2, pp. 203-214, 2011.

[32] L. Meng, X. He, W. Zhu et al., "TLR3 and TLR7 modulate IgE production in antigen induced pulmonary inflammation via influencing IL-4 expression in immune organs," PLOS ONE, vol. 6, no. 2, Article ID e17252, 2011. 
[33] B. Frotscher, K. Anton, and M. Worm, "Inhibition of IgE production by the imidazoquinoline resiquimod in nonallergic and allergic donors," Journal of Investigative Dermatology, vol. 119, no. 5, pp. 1059-1064, 2002.

[34] E. Shen, L. Lu, and C. Wu, "TLR7/8 ligand, R-848, inhibits IgE synthesis by acting directly on B lymphocytes," Scandinavian Journal of Immunology, vol. 67, no. 6, pp. 560-568, 2008.

[35] F. Brugnolo, S. Sampognaro, F. Liotta et al., "The novel synthetic immune response modifier R-848 (Resiquimod) shifts human allergen-specific $\mathrm{CD}_{4}{ }^{+} \mathrm{T}_{H} 2$ lymphocytes into IFN- $\gamma$-producing cells," Journal of Allergy and Clinical Immunology, vol. 111, no. 2, pp. 380-388, 2003.

[36] M. Wills-Karp, J. Luyimbazi, X. Xu et al., "Interleukin-13: central mediator of allergic asthma," Science, vol. 282, no. 5397, pp. 2258-2261, 1998.

[37] F. Grela, A. Aumeunier, E. Bardel et al., "The TLR7 agonist R848 alleviates allergic inflammation by targeting invariant NKT cells to produce IFN- $\gamma$," The Journal of Immunology, vol. 186, no. 1, pp. 284-290, 2011.

[38] Q. Du, L.-F. Zhou, Z. Chen, X.-Y. Gu, M. Huang, and K.-S. Yin, "Imiquimod, a toll-like receptor 7 ligand, inhibits airway remodelling in a murine model of chronic asthma," Clinical and Experimental Pharmacology and Physiology, vol. 36, no. 1, pp. 43-48, 2009.

[39] P. Camateros, M. Tamaoka, M. Hassan et al., "Chronic asthmainduced airway remodeling is prevented by toll-like receptor7/8 ligand S28463," American Journal of Respiratory and Critical Care Medicine, vol. 175, no. 12, pp. 1241-1249, 2007.

[40] S. W. Lee, D. R. Kim, T. J. Kim et al., "The association of downregulated toll-like receptor 4 expression with airflow limitation and emphysema in smokers," Respiratory Research, vol. 13, article106, 2012.

[41] M. Werner, R. Topp, K. Wimmer et al., "TLR4 gene variants modify endotoxin effects on asthma," Journal of Allergy and Clinical Immunology, vol. 112, no. 2, pp. 323-330, 2003.

[42] Q. Zhang, F. H. Qian, L. F. Zhou et al., "Polymorphisms in toll-like receptor 4 gene are associated with asthma severity but not susceptibility in a Chinese Han population," Journal of Investigational Allergology and Clinical Immunology, vol. 21, no. 5, pp. 370-377, 2011.

[43] K. Tizaoui, W. Kaabachi, K. Hamzaoui, and A. Hamzaoui, "Association of single nucleotide polymorphisms in Toll-like receptor genes with asthma risk: a systematic review and metaanalysis," Allergy, Asthma \& Immunology Research, vol. 7, no. 2, pp. 130-140, 2015.

[44] E. M. M. Klaassen, B. E. J. T. Thönissen, G. van Eys, E. Dompeling, and Q. Jöbsis, "A systematic review of CD14 and toll-like receptors in relation to asthma in Caucasian children," Allergy, Asthma and Clinical Immunology, vol. 9, article 10, 2013.

[45] F. H. Qian, Q. Zhang, L. F. Zhou, G. F. Jin, J. L. Bai, and K. S. Yin, "Polymorphisms in the toll-like receptor2 subfamily and risk of asthma: a case-control analysis in a Chinese population," Journal of Investigational Allergology and Clinical Immunology, vol. 20, no. 4, pp. 340-346, 2010.

[46] A. Heinzmann, M. Brugger, S. Bierbaum, B. Mailaparambil, M. V. Kopp, and K. Strauch, "Joint influences of Acidic-Mammalian-Chitinase with Interleukin-4 and Toll-like receptor-10 with Interleukin-13 in the genetics of asthma," Pediatric Allergy and Immunology, vol. 21, no. 4, part 2, pp. e679-e686, 2010.

[47] R. Lazarus, W. T. Klimecki, B. A. Raby et al., "Single-nucleotide polymorphisms in the Toll-like receptor 9 gene (TLR9): frequencies, pairwise linkage disequilibrium, and haplotypes in three U.S. ethnic groups and exploratory case-control disease association studies," Genomics, vol. 81, no. 1, pp. 85-91, 2003.

[48] M. S. D. Kormann, M. Depner, D. Hartl et al., "Toll-like receptor heterodimer variants protect from childhood asthma," Journal of Allergy and Clinical Immunology, vol. 122, no. 1, pp. 86-e8, 2008.

[49] T. V. Grissell, H. Powell, D. R. Shafren et al., "Interleukin-10 gene expression in acute virus-induced asthma," American Journal of Respiratory and Critical Care Medicine, vol. 172, no. 4, pp. 433439, 2005.

[50] P. A. B. Wark, S. L. Johnston, I. Moric, J. L. Simpson, M. J. Hensley, and P. G. Gibson, "Neutrophil degranulation and cell lysis is associated with clinical severity in virus-induced asthma," European Respiratory Journal, vol. 19, no. 1, pp. 68-75, 2002.

[51] H. Rupani, R. T. Martinez-Nunez, P. Dennison et al., "Toll-like receptor 7 is reduced in severe asthma and linked to altered microRNA profile," American Journal of Respiratoryand Critical Care Medicine, 2016.

[52] M. Contoli, S. D. Message, V. Laza-Stanca et al., "Role of deficient type III interferon- $\lambda$ production in asthma exacerbations," Nature Medicine, vol. 12, no. 9, pp. 1023-1026, 2006.

[53] A. Sykes, M. R. Edwards, J. MacIntyre et al., "Rhinovirus 16induced IFN- $\alpha$ and IFN- $\beta$ are deficient in bronchoalveolar lavage cells in asthmatic patients," Journal of Allergy and Clinical Immunology, vol. 129, no. 6, pp. 1506-1514, 2012.

[54] A. L. Pritchard, O. J. White, J. G. Burel, M. L. Carroll, S. Phipps, and J. W. Upham, "Asthma is associated with multiple alterations in anti-viral innate signalling pathways," PLoS ONE, vol. 9, no. 9, Article ID e106501, 2014.

[55] M. Matsumoto, H. Oshiumi, and T. Seya, "Antiviral responses induced by the TLR3 pathway," Reviews in Medical Virology, vol. 21, no. 2, pp. 67-77, 2011.

[56] K. S. Parsons, A. C. Hsu, and P. A. B. Wark, “TLR3 and MDA5 signalling, although not expression, is impaired in asthmatic epithelial cells in response to rhinovirus infection," Clinical and Experimental Allergy, vol. 44, no. 1, pp. 91-101, 2014.

[57] R. H. Xu, E. B. Wong, D. Rubio et al., "Sequential activation of two pathogen-sensing pathways required for Type I interferon expression and resistance to an acute DNA virus infection," Immunity, vol. 43, no. 6, pp. 1148-1159, 2015.

[58] D. Tworek, S. G. Smith, B. M. Salter et al., "Interleukin 25 receptor expression on airwaydendritic cells after allergen challenge in asthmatic subjects," American Journal of Respiratory and Critical Care Medicine, 2015.

[59] M. J. Duechs, C. Tilp, C. Tomsic, F. Gantner, and K. J. Erb, "Development of a novel severe triple allergen asthma model in mice which is resistant to dexamethasone and partially resistant to TLR7 and TLR9 agonist treatment," PLOS ONE, vol. 9, no. 3, Article ID e91223, 2014.

[60] J. N. Kline, T. J. Waldschmidt, T. R. Businga et al., "Modulation of airway inflammation by CpG oligodeoxynucleotides in a murine model of asthma," The Journal of Immunology, vol. 160, no. 6, pp. 2555-2559, 1998.

[61] A. Lhrmann, T. Tschernig, H. von der Leyen, M. Hecker, R. Pabst, and A. H. Wagner, "Decoy oligodeoxynucleotide against STAT transcription factors decreases allergic inflammation in a rat asthma model," Experimental Lung Research, vol. 36, no. 2, pp. 85-93, 2010.

[62] V. V. Jain, K. Kitagaki, T. Businga et al., "CpG-oligodeoxynucleotides inhibit airway remodeling in a murine model of chronic 
asthma," Journal of Allergy and Clinical Immunology, vol. 110, no. 6, pp. 867-872, 2002.

[63] S. Y. Lee, J. Y. Cho, M. Miller et al., "Immunostimulatory DNA inhibits allergen-induced peribronchial angiogenesis in mice," Journal of Allergy and Clinical Immunology, vol. 117, no. 3, pp. 597-603, 2006.

[64] D.-J. Chiang, Y.-L. Ye, W.-L. Chen, Y.-L. Lee, N.-Y. Hsu, and B.-L. Chiang, "Ribavirin or CpG DNA sequence-modulated dendritic cells decrease the IgE level and airway inflammation," American Journal of Respiratory and Critical Care Medicine, vol. 168, no. 5, pp. 575-580, 2003.

[65] S. Ashino, D. Wakita, Y. Zhang, K. Chamoto, H. Kitamura, and T. Nishimura, "CpG-ODN inhibits airway inflammation at effector phase through down-regulation of antigen-specific Th2-cell migration into lung," International Immunology, vol. 20, no. 2, pp. 259-266, 2008.

[66] A. Fedulov, E. Silverman, Y. Xiang, A. Leme, and L. Kobzik, "Immunostimulatory $\mathrm{CpG}$ oligonucleotides abrogate allergic susceptibility in a murine model of maternal asthma transmission," The Journal of Immunology, vol. 175, no. 7, pp. 4292-4300, 2005.

[67] Z. Aryan, S. T. Holgate, D. Radzioch, and N. Rezaei, "A new era of targeting the ancient gatekeepers of the immune system: tolllike agonists in the treatment of allergic rhinitis and asthma," International Archives of Allergy and Immunology, vol. 164, no. 1, pp. 46-63, 2014.

[68] C. M. Trujillo-Vargas, J. R. Ramirez-Pineda, A. Palmetshofer et al., "Mice vaccinated with allergen-pulsed myeloid dendritic cells are not protected from developing allergen-induced Th2 responses," International Archives of Allergy and Immunology, vol. 137, no. 3, pp. 219-228, 2005.

[69] S. Kapoor, L. Geng, A. Feldman, and H. Jyonouchi, "Characterization of severe asthma (SA) in children of inner-city of Newark: altered responses to recall antigens and a panel of agonists of Toll-like receptors (TLR)," Journal of Allergy and Clinical Immunology, vol. 127, article AB61, 2011.

[70] M. Tamm, D. H. Richards, B. Beghé, and L. Fabbri, "Inhaled corticosteroid and long-acting $\beta 2$-agonist pharmacological profiles: effective asthma therapy in practice," Respiratory Medicine, vol. 106, no. 1, pp. S9-S19, 2012.

[71] C. Xirakia, O. Koltsida, A. Stavropoulos et al., “Toll-like receptor 7-triggered immune response in the lung mediates acute and long-lasting suppression of experimental asthma," American Journal of Respiratory and Critical Care Medicine, vol. 181, no. 11, pp. 1207-1216, 2010.

[72] J. Moisan, P. Camateros, T. Thuraisingam et al., "TLR7 ligand prevents allergen-induced airway hyperresponsiveness and eosinophilia in allergic asthma by a MYD88-dependent and MK2-independent pathway," American Journal of PhysiologyLung Cellular and Molecular Physiology, vol. 290, no. 5, pp. L987-L995, 2006.

[73] D. Quarcoo, S. Weixler, R. A. Joachim et al., "Resiquimod, a new immune response modifier from the family of imidazoquinolinamines, inhibits allergen-induced Th2 responses, airway inflammation and airway hyper-reactivity in mice," Clinical and Experimental Allergy, vol. 34, no. 8, pp. 1314-1320, 2004.

[74] K. Ito, K. F. Chung, and I. M. Adcock, "Update on glucocorticoid action and resistance," Journal of Allergy and Clinical Immunology, vol. 117, no. 3, pp. 522-543, 2006.
[75] E. Chun, S.-H. Lee, S.-Y. Lee et al., “Toll-like receptor expression on peripheral blood mononuclear cells in asthmatics; implications for asthma management," Journal of Clinical Immunology, vol. 30, no. 3, pp. 459-464, 2010.

[76] E. Pace, C. Di Sano, M. Ferraro et al., "Budesonide increases TLR4 and TLR2 expression in Treg lymphocytes of allergic asthmatics," Pulmonary Pharmacology and Therapeutics, vol. 32, pp. 93-100, 2015. 


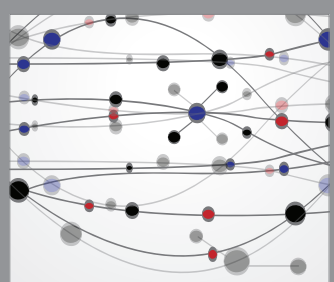

The Scientific World Journal
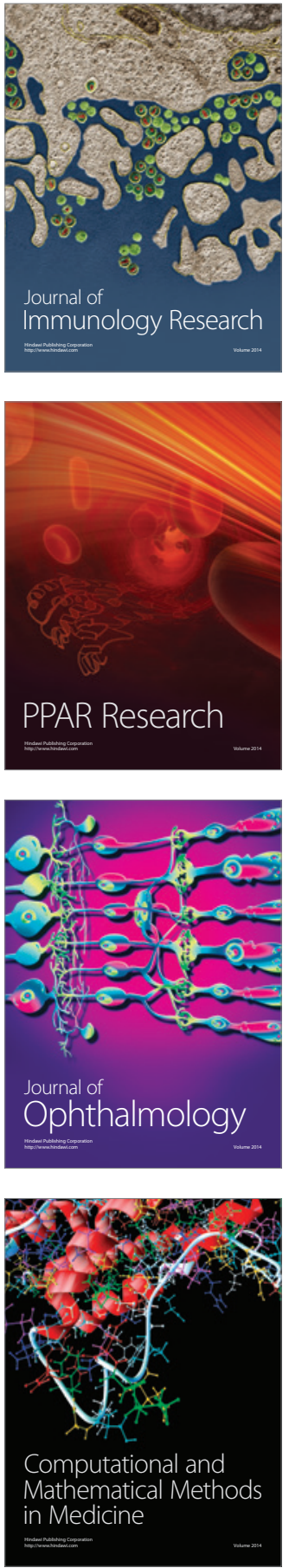

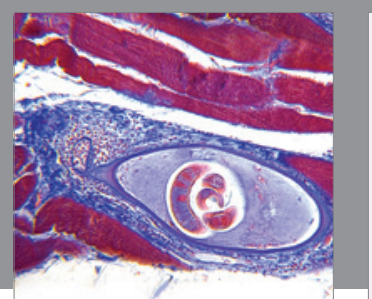

Gastroenterology Research and Practice

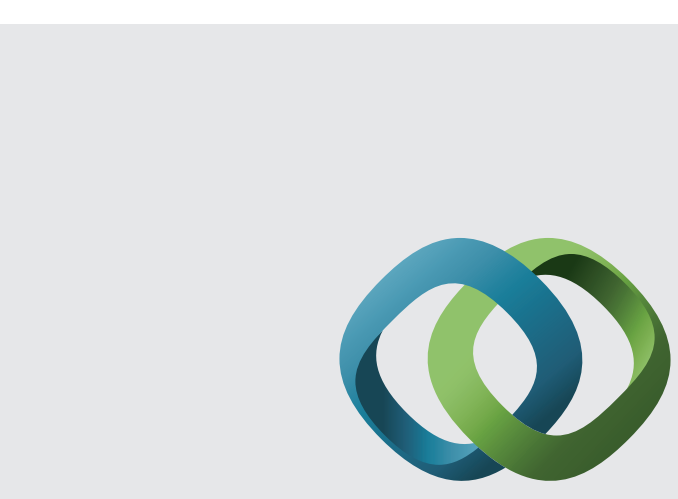

\section{Hindawi}

Submit your manuscripts at

http://www.hindawi.com
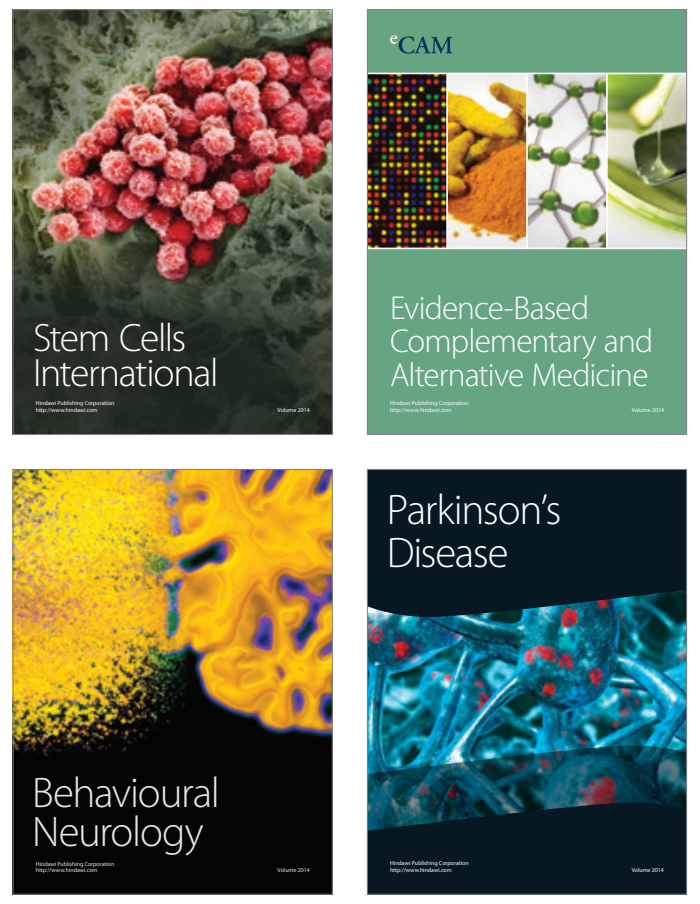
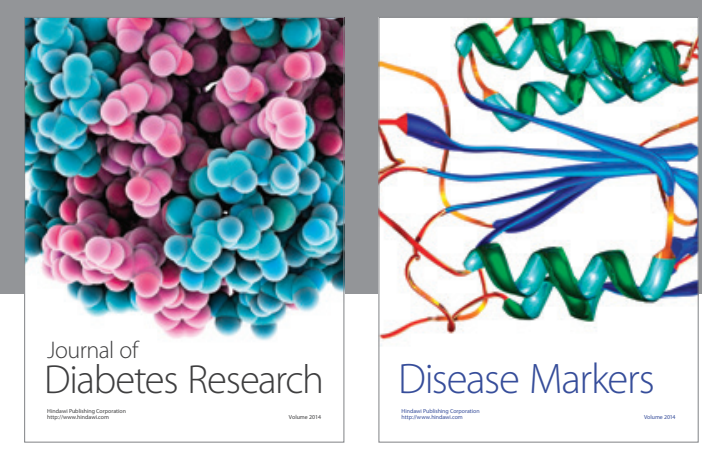

Disease Markers
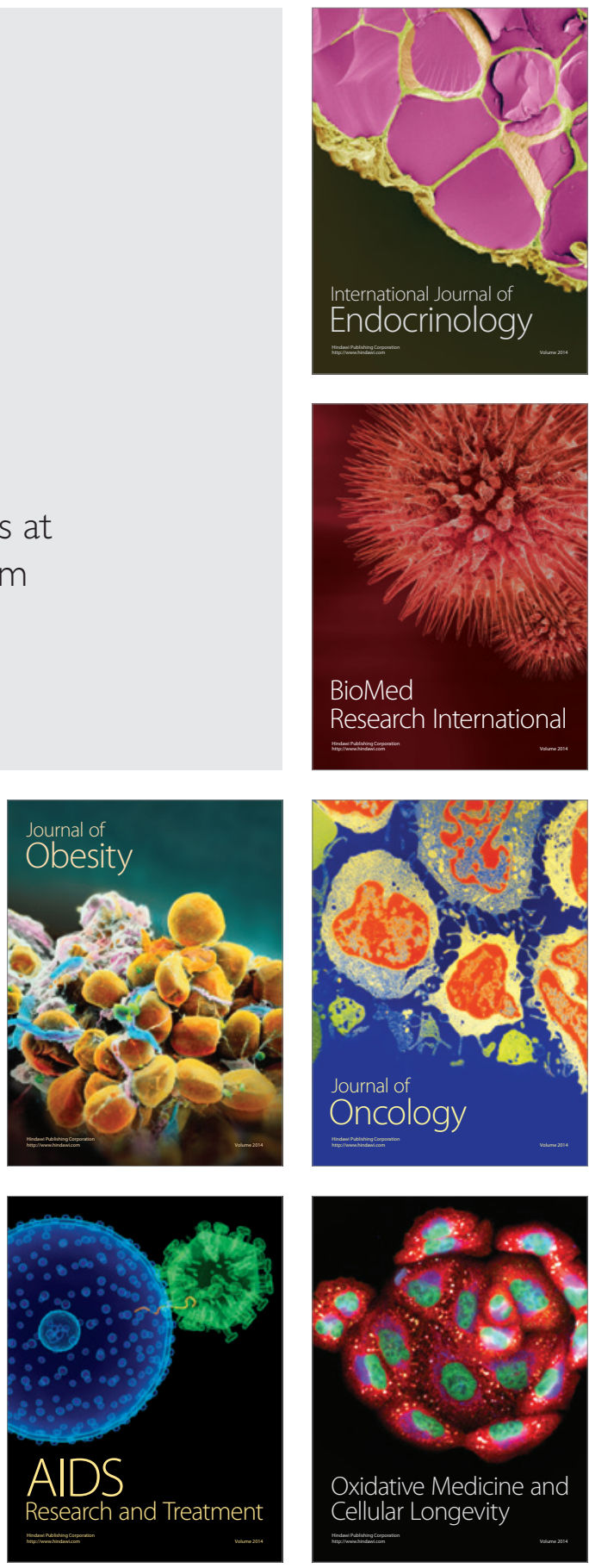